\title{
A proposal to organize i2-EPR (International Institute for Exploring Physics with Reality)
}

Chandrasekhar Roychoudhuri

Chandrasekhar Roychoudhuri, "A proposal to organize i2-EPR (International Institute for Exploring Physics with Reality)," Proc. SPIE 9665, Tenth International Topical Meeting on Education and Training in Optics and Photonics, 96650C (3 June 2007); doi: 10.1117/12.2207529

SPIE Event: Tenth International Topical Meeting on Education and Training in Optics and Photonics, 2007, Ottawa, Ontario, Canada 


\title{
Draft-1 (July 30, 2007) \\ A proposal to organize
}

\author{
$i^{2}$-EPR \\ (International Institute for Exploring Physics with Reality)
}

How can we promote discovery of actual realities in nature driven by cosmic logic rather than staying limited to invention of realities that are esthetically pleasing to our human logic?

\section{by}

\section{Prof. Chandrasekhar Roychoudhuri}

Femto Macro Continuum

7 Fieldstone Drive, Storrs, CT 06268, USA

$\&$

University of Connecticut

Photonics Lab., Department of Physics

54 Ahern Lane, Storrs, CT 06269-5192, USA 


\title{
Executive Summary
}

\author{
Mission
}

-1. Research: Our objective is to discover, visualize, understand and some times gainfully emulate (to advance our technologies) the real physical processes behind diverse interactions that are at the root of incessant cosmic and biospheric evolutions. Our focus is on discovering actual realities in nature driven by cosmic logic rather than inventing the ones that are aesthetically pleasing to our human logic. Our responsibility is to facilitate continuous evolution in our epistemology of modeling natural phenomena simultaneously exploiting the current tools of reductionism and emergence-ism that are helping us to discover rules of interactions between stable but simple (some times irreducible) entities and between complex assemblies that emerge from simpler entities.

- 2. Education: Promote an educational philosophy that encourages the students to persistently enquire to visualize and understand the processes behind all interactions in nature while being conscious of their scientific epistemology. Everything that we "see" is nothing but a creative interpretation of the chain of transformations experienced by the sensor (or assemblies of sensors) that we use to observe nature. Science has so far formulated an array of working rules to model nature none of which can be declared as inviolable laws as yet. We have generated several "solved" jig-saw-puzzles which are not yet unifiable into one coherent puzzle to map the indivisible cosmic system.

- 3. Outreach: Organize local and international seminars and conferences disseminating (i) the results of research, and (ii) the evolving \& effective model of research (scientific epistemology).

-4. Economic wellbeing: Disseminate new technology innovation potentials to attract enhanced economic support through proper local channels.

\section{Structure}

- 1. A Virtual Global Institute: A limited number of agreeable institutions from several countries will organize their own local centers (i-EPR) at their own cost, congruent with their institutional mission and local governance. The local centers will adhere to the core vision and mission of the i-square-EPR constituting representatives from the local centers.

- 2. Organizational principles: The interactions between the local centers should be driven by (i) symbiosis, (ii) synergy and (iii) food-chain (iv) eco-driven (v) competitions, which are behind the successful and sustainable evolution of our organic biospheric system.

\section{Approach}

- Nature being a creative system engineer we should be humble but creative reverse engineers to extract the working rules behind natural processes in nature and aid to technology advancements that are congruent with our sustainable evolution, for we need a long long time to understand the purpose of the cosmic evolution and our role in it. 


\section{Proposer's demonstrated organizational successes}

\section{Acknowledgements}

1. OSA \& SPIE - for providing the platforms to raise the following question even though the main stream physics community believes that it is no longer an issue!

2. NSG \& OTRR - for financial sponsorship for related publication and conferences.

The Nature of Light: What Are Photons?

Recent references

2003

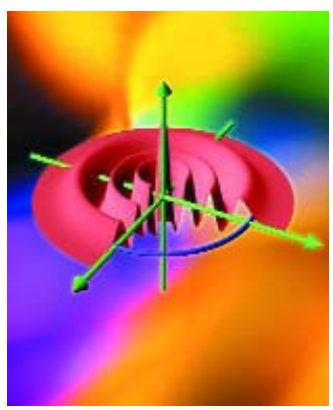

Optics and

Photonics News

Special Issue;

October 2003

Guest Editors:

C. Roychoudhuri and R.

Roy
2005

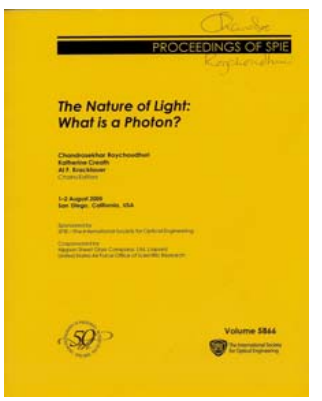

SPIE Proc.

Vol.5866

Conference Editors:

C. Roychoudhuri, K.

Creath \& A.

Kracklauer
2007

The Nature of Light: What

is a Photon? - II

Part of the SPIE International

26-30 August 2007 • San Diego

Conference Chairs: Chandra

Roychoudhuri, Univ. of Connecticut;

Al F. Kracklauer, Consultant

(Germany) Katherine Creath,

Optineering and Univ. of Arizona

SPIE Proc. 6664 


\section{Background \& Justification}

Proc. of SPIE Vol. 9665 96650C-4 


\section{Why seek reality?}

\section{Manifest and emergent material universe is real and causal. It is subtle, complex and elusive, but it is neither mystical nor an a-causal illusion!}

We need to understand the real physical processes behind the emergence of both the irreducibly stable elementary particles as well as the most complex systems out of these elementary particles. Then we can emulate the processes to assure our sustainable evolution, which will give us time to understand the meaning \& purpose of the universe and our role in it !

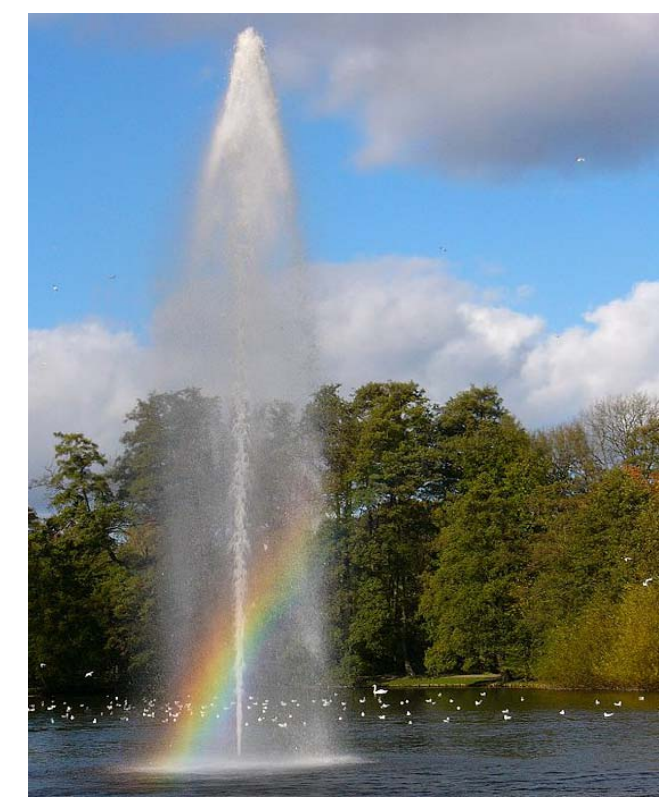

Photons do not have any colors! Neither are the water droplets colored inside the fountain! [Photo taken from the web.]
If you go inside the fountain, you will not find the rainbow.

Yet, it is a reality in nature \& not an illusion,. It can be observed only by color-interpreting frequency sensitive sensors and only at the focal plane of an imaging system looking at the fountain and only when the Sun is behind the imaging sensor (observer).

There is no real rainbow inside the fountain. That is why nobody goes to dig out the "pot of gold at the end of the rainbow".

The emergence of the mystical beauty of a rainbows is elusive but causal, and definitely not an illusion! Understanding the processes behind its formation help us understand the local weather conditions, besides enjoying its esthetic beauty, while advancing the optical sciences by validating a good number of optical principles through a single emergent phenomenon! 


\section{Why another research organization?}

- Deviation from Reality: While technologies have been advancing successfully, the fundamental understanding of actual processes in nature has not only stalled for almost 50 years, it has seriously deviated into justifying mysticism by promoting non-causality, non-locality, teleportation, multi-universe, etc., which are hard to experimentally invalidate.

- "Nobody understands Quantum Mechanics" even though it works!: If nobody understands QM even after 82 years' of developing the formalism and its successful predictions, then we ought to acknowledge that there is some serious flaw with our epistemology to map or understand nature. Interpretation of QM and its successes to predict observations are not mutually congruent in seeking actual reality in nature. Classical physics, by being faithfully congruent to seeking reality in nature, gave birth to QM. But QM has failed to give birth to its progeny due its assumption that it is "complete".

- From Geocentric to Homocentric: After overriding Ptolemy's Geocentric vision, we are back on imposing Homocentric vision on all the enquiring minds by re-directing them to model nature as dictated by our human mathematical logic, instead of seeking actual reality. Being stifled by the quantum philosophy, we are emulating the 'invention culture' from our technological successes and imposing that on to physics. We are now deeply engaged in inventing realities rather than discovering the actual realities behind nature's evolutionary processes.

- Cosmic vs. Human logic \& CC-LC-ER epistemology : Our cumulative experience indicates that nature's evolutionary processes consist of logical patterns \& organizations. Although only partial, our consistent successes have been based on applying limited human logic to reach out to unknown cosmic logic. Our efforts to refine and elevate human logic to possible cosmic logic has been advancing based on the model of CC-LC - pro-actively extend Conceptual Continuity among as many diverse natural phenomena as possible by imposing Logical Congruence. Our belief in this CC-LC epistemology and intuitive faith in one continuous and logically functioning universe have been paying off enormously. Current physics has been developed based essentially on reductionism, matter into elementary particles and radiations into photons. We have neglected to incorporate formalism that articulates emergence of properties and rules of interactions in and between complex systems that constitute our regular observable universe. We now need to add another iterative feed back loop of Reductionism and Emergence-ism, CC-LC-ER epistemology, which should enhance the unifiability of our separate models or maps of nature.

- "Did the tree fall?": We now know that all advanced specie contain large assembly of diverse symbiotic and some nonsymbiotic organisms. Our 10 trillion-cell human body carries 100 trillion diverse symbiotic microbes. To what extent our human thought processes are influenced by the convergent-divergent self interests of these two groups of co-habiting cells? In the absence of any human being in a forest, the fallen trees are properly taken care of by trillions of local microbes and more "advanced" organisms! Homocentric philosophies are counter to our healthy evolution.

- Conscious Epistemology: Do we really understand how our thinking process evolves? Can we identify \& understand how all the factors that influence our conclusions after collating diverse observations? Epistemological awareness is crucial for us. 


\section{Some organized global minorities are restless to bring changes!}

\section{They are catalyzing changes in scientific epistemology!}

- Status-quo of the main stream: Existing basic (working) theories must not be challenged! All the main stream institutions and journals around the globe pro-actively rejects that progress in physics is being stifled by accepting that all the current theories are correct as they are within their respective domains. They agree that the universe is one continuum. Yet they ignore the message of failure over many decades of intensive attempts to unify the separate little maps ("solved" jig-saw-puzzles) into one coherent structure.

- NPA: Founded in 1994, this group has just finished their $12^{\text {th }}$ conference at the University of Connecticut. NPA nurtures the enquiring minds of their members who are seeking reality in nature

• "Nature of Light: What Are Photons?": In 2003 we initiated this organized questioning about photon, first, by publishing a special issue on the topic through the OSA-OPN (Optics \& Photonics News) issue of October. Then, in 2005 we organized an international conference on the same topic under the auspices of the SPIE Annual Conference with explicit intention of nurturing out-of-box thinking about photon and their consequences in physics and engineering. It has now become a well-accepted biannual conference; the $2^{\text {nd }}$ conference is about to be held in San Diego during Aug.26-27.

- Individual well-known authors challenging the status-quo: The following recent books are beginning to draw attention to the lack of progress in physics: (i) Roger Penrose, "The Road to Reality" (2004) (ii) Nobel Laureate Robert Laughlin, "A Different Universe" (2005), (iii) Lee Smolin, "The trouble with Physics" (2006).

- Individual web sites: Many individuals, rejected and disenchanted by the main stream academia, have opened their personal websites explaining their alternate opinions about all the currently accepted physics theories.

- Role of i-square-EPR: We hope to synergize the energy of all these brave souls by facilitating their collaborative interactions through a virtual global institution respecting individual independence \& freedom.. 


\section{Proposed philosophy behind $i^{2}$-EPR}

- Vision: The grand vision is to nurture the emergence of a global culture that accepts the responsibility of pro-active conscious designing of our future as the basis for our sustainable evolution within the biosphere.

The scientific vision is to re-establish physics as a discipline that is firmly engaged to discover the objective reality of nature as it happens, rather than inventing mathematically self-congruent model of homocentric reality and impose that on the nature. The idea is to continuously explore visualize and imagine the actual processes that are going on behind all the various interactions leading to incessant evolution of the biospheric and the cosmo-spheric evolutions.

Pragmatic dimension: Sustainable evolution is the prime desire evident form the genetic coding of all the specie. We need continuous expansion of our knowledge of various organizational structures in nature to develop newer technological tools products and engineering for our well being that are congruent with the rules of interactions in nature. Thus looking at nature as a creative system engineer and behaving ourselves as reverse engineers to emulate nature's processes is the best successful model for us. Science engineering economics and politics are inseparable social enterprises for our survival.

- Spiritual dimension: What are the meaning and purpose of our lives? Can we control and design our fate? The emergence of the spiritual dimension out of our scientific (reverse engineer) thinking started taking shape as we slowly started to appreciate the ever harmonizing organizations behind diverse processes in nature. We realized the dialectical state of our fate in nature - we are simultaneously the masters of our well being and yet insignificant 'pawn' in the evolutionary scheme of things in the "infinite" cosmos.

- Approach, the p-cubed methodology: The ultimate purpose of the scientific enterprise is to become the best possible creative reverse engineers, extract the organizational rules behind ongoing processes in nature and then emulate them as beneficial technologies that are congruent with our sustainable evolution, since we need a long long time to understand the real purpose of the cosmic evolution and our role in it. Nature is our best peer. Sustainable engineering activities will keep us anchored to nature's reality. 


\title{
All major organized human enterprises must define its "pragmatic guiding star", an infallible vision
}

\begin{abstract}
- Prime directive: Live and let live. Today we know enough to destroy the biosphere and hence ourselves, and yet we are not knowledgeable enough to model and nurture the biosphere in every scientific detail to assure our co-dependent survival. And we are very far from becoming space-farer en-masse to migrate to another earth-like planet.

- Nature is a creative system engineer: Irrespective of our diverse spiritual believes, we have been successfully evolving for millennia in human form as reverse engineers by developing technologies (from discovering fire and rockets to medicine delivering nano particles in blood) by understanding \& emulating the working processes and the underlying working rules behind diverse natural phenomena.
\end{abstract}

- Science-technology-economy-politics (STEP) and sustainable evolution: In every step of our evolution from primitive to modern days, STEP functions have always necessarily been and always will be inseparable from each other because the sustainable evolution is our prime directive and embedded in our genes.

- STEP leads to enquiry based spiritualism, or becomes STEPS: Healthy spirituality, or the enquiry about meaning, purpose and future vision of life, individualistic or biospheric, evolves through systematic observation of nature's evolutionary processes. We find the organizational patterns and working rules in them and then use those rules intelligently for our personal evolution congruent with the collective evolution.

- Like all social enterprises, i-sqaure-EPR will have a focused objective: It is scientific - keep on mapping nature's interaction processes with increasingly higher level of integrations of wide ranging natural phenomena. But our "guiding star" is the aspiration to understand the meaning \& purpose of the cosmic evolution and our role in it by being a facilitator to sustainable evolution by promoting technology innovations as creative-reverse-engineers! 


\section{Proposed structure of $i^{2}$-EPR}

- A Virtual Global Institute: A limited number of agreeable institutions from several countries will organize their own local institutes (i-EPR) at their own cost, congruent with their institutional mission and local governance. All these i-EPR will adhere to the core vision of the i-sqaure-EPR. The international virtual global center, composed of representatives from all the i-EPR's, will have the role of a facilitator. Because funding is local, all the research activities will reside in the local institutes. The international body will only facilitate the coordination of the short- and long-term research programs to be complementary to each other to maximize the collective speed and productivity of all.

- Organizational principle: We shall emulate to the best of our knowledge the organizational principles behind the best organically evolving system to our knowledge - the collective evolution of lives in the biosphere. They are driven by (i) symbiosis, (ii) synergy and (iii) food-chain (iv) eco-driven (v) competition. It is not pure "survival of the fittest". The organizational principles must be pro-actively congruent towards empowerment of the local centers and individual researchers and rules must be designed for guiding rather than controlling and stifling activities. 


\section{Why virtual and why global?}

- Dichotomy of academic freedom and strangulation: All important human social enterprises are meant to be organized and managed to maximize the collective benefit to the entire society empowerment of all by allowing development of knew knowledge and their dissemination. Unfortunately, self-interests of well organized groups have always taken precedence. New knowledge and information are used to maintain the privileged position and the associated benefits. The livelihood (economic survival) depends upon being able to "fit into" the main stream. "Acceptance" and "Group-think" are pragmatism! [See Smolin's book]. New scientific concepts that can potentially challenge those currently in main stream, can challenge the structural power and access to limited Government funds from those who are in decision making positions including all those who rely on the benefits percolating down from these power positions. Thus, "nobody understands quantum mechanics" is not sufficient reason to challenge the interpretations of quantum mechanics. Teleportation and Quantum Computing are drawing enormous funds!

- Why Global? Avoid strangulation: Only properly funded institutions can attract brilliant minds. Academic institutions are still the biggest draw of minds. Thus, a university would be the best place to start such a research institute. Unfortunately, an out-of-box research center based in a single university could face strangulation at birth! A globally distributed organization will have a much higher probability of succeeding in its eventual mission.

- Why Global? Magnitude and weight of the proposed research problem : The proposed research problem is enormously important and burdensome. It requires global attention and global participation. Midwifery for the birth of the progeny of Quantum Mechanics (QM) will require intensive attention from many creative and unorthodox scientists just as were required for the birth of QM out of Classical Physics. 


\section{Building the case for CC-LC-ER epistemology for i-square-EPR activities}

- Physics is synonymous with seeking Conceptual Continuity (CC) among diverse observed phenomena by iteratively and creatively constructing Logical Congruence ( $L C$ ) among them all to find a higher level of organization leading to coherent maps of nature.

- But to understand the structure of particles and the interaction processes behind the observed results to visualize elusive realities, we must integrate Emergencism and Reductionism to CC-LC-thinking to develop CC-LC-ER epistemology. This will help us understand the inner structures of the interactants and the resultant products) by enquiring and distinguishing for their new Emergent properties from those they possess when they are Reduced to more fundamental constituent elements. Emergencism and Reductionism are inseparable complementary modes of enquiries. 


\section{Understanding the subtleties behind observations before applying CC-LC-ER epistemology}

Real world is really real if we spend time to find the conceptual continuity amongst our diverse observations by imposing logical congruency through all ! Our lack of fathoming the real processes undergoing in the micro universe may appear elusive, but it does not mean that the real universe is non-causal, non-local and mystical! Emergence of complex properties can also be logically mapped.

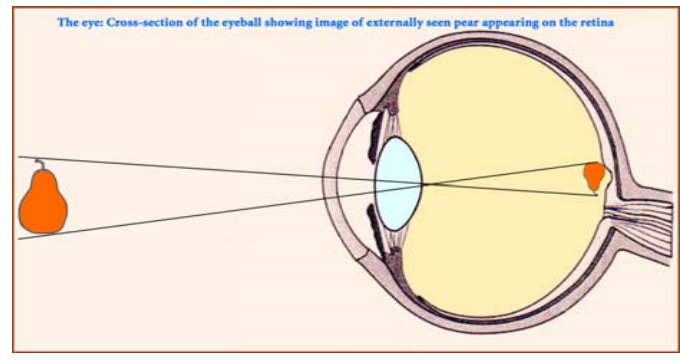

Human neural-nets reconstruct the reality out of the inverted digital image formed on the rods and cones of our retina.

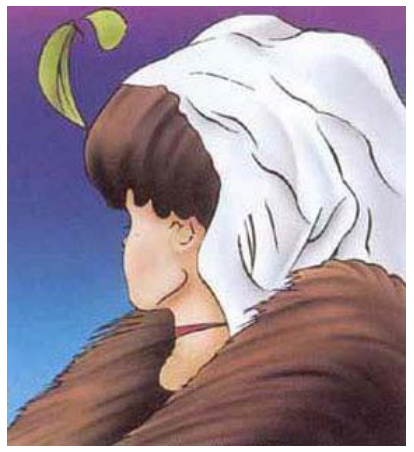

Elusive but real. Choose between young or old woman by differentiating chin vs. nose.

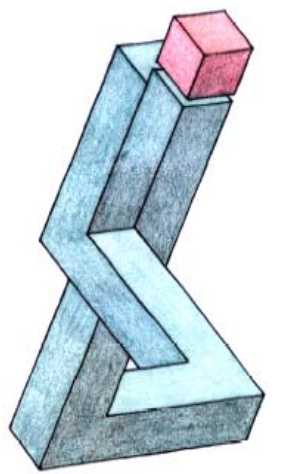

A real illusion. Looks real but impossible to construct in reality.

Personally, I really am not totally objective about the outside universe. I simply cannot be! I "see" what is really functionally important for my survival in the real world with my limited number of sensors. My thinking and interpretations are also colored by my genetic endowment, 100 trillion symbiotic microbes and bacteria, my family and social training and my personal knowledge and ego driven by my own successes and failures! Objectivity of our interpretations have to be refined by repeated application of CC-LC-ER epistemology. [Above pictures are taken from the web.] 


\section{Mathematical congruency and visual symmetry \& elegance are insufficient guides to extract nature's objective reality}

CC-LC-ER epistemology is a critical tool!

Consider two examples:

1. Pythagoras' theorem and

2. Ptolemy's Geocentric planetary model 


\section{Pythagoras' single quadratic relation can be replaced by a pair of linear relations}

Consider Pythagoras' theorem

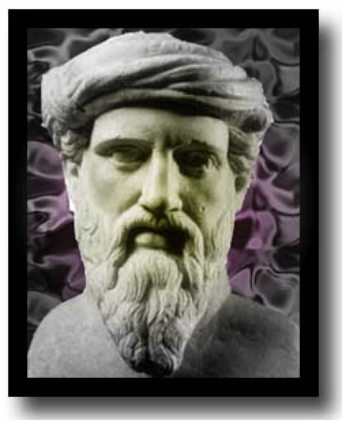

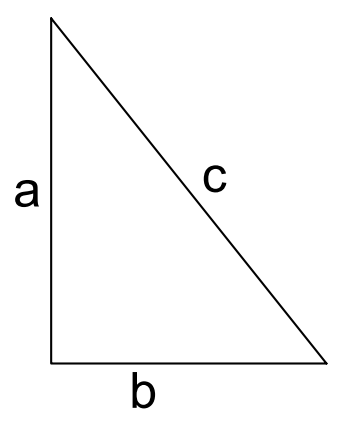

$c^{2}=a^{2}+b^{2}$
Compare with my modified theorem from the $7^{\text {th }}$ grade home work:

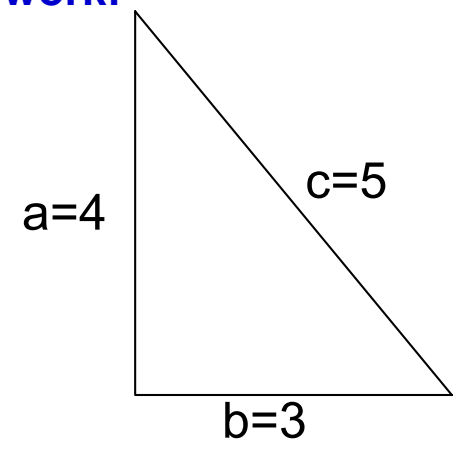

vs. $c=2 a-b$ where $\frac{a}{b}=\frac{4}{3}$

Mathematically they are equivalent to each other ! 


\section{But, Pythagoras' relation aids the visualization of the logic behind its success. My linear relations work but fail to aid the visualization.}

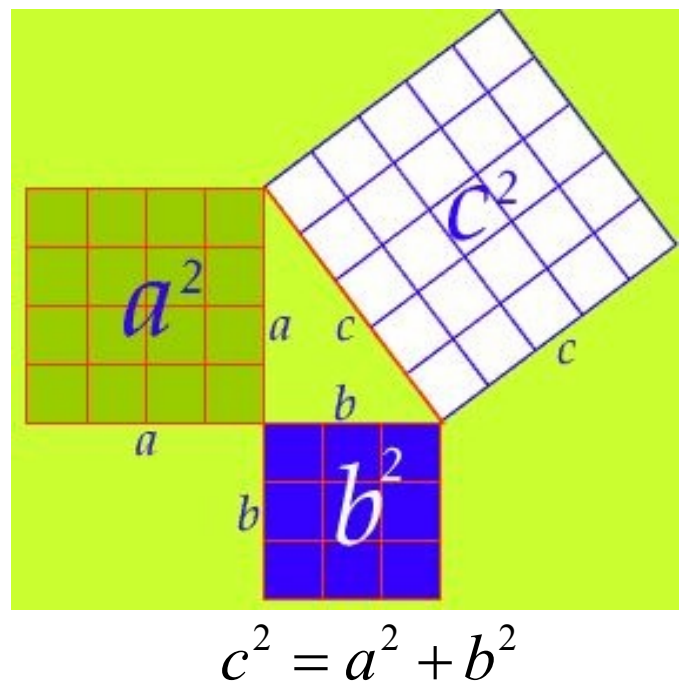

The total number of unit squares on the hypotenuse is exactly equal to the some of those on the other two sides. [The picture is taken from the web.]
My relations do not directly give any insight into the physical reason of correctness.

It just works!

$$
\begin{aligned}
& c=2 a-b \text { with } \frac{a}{b}=\frac{4}{3} \\
& c^{2}=4 a^{2}+b^{2}-4 a b \\
& =4 a^{2}+b^{2}-4 a \cdot \frac{3}{4} a \\
& c^{2}=a^{2}+b^{2}
\end{aligned}
$$




\section{Revivability of Ptolemy's Geocentric planetary model !}

\section{Even visualization is not enough to guide us into discovering nature's reality}

Many different human logic (mathematics) can solve the same problem different ways. Ptolemy's Geocentric planetary model can be successfully updated with nine different "epicycles" for each of the nine planets. This is far fewer "free parameters" than most of the recent "successful" string theories require!

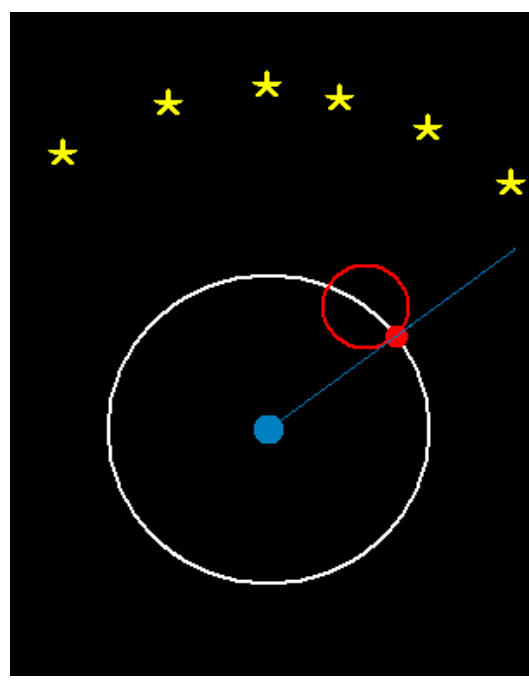

Ptolemy

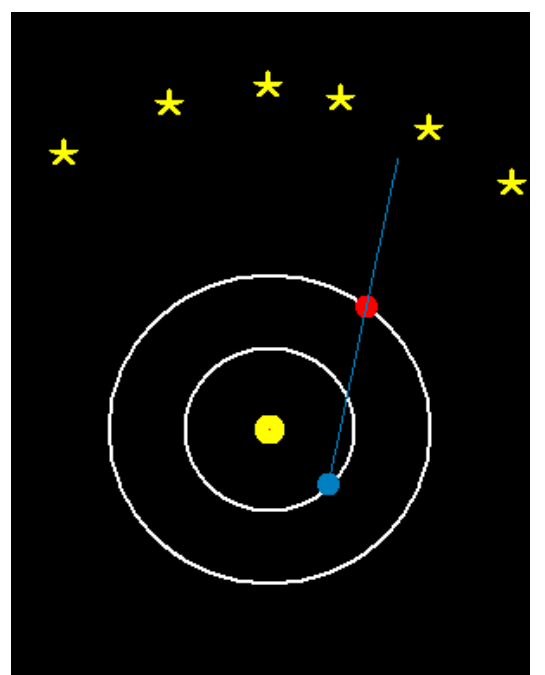

Pictures are from the web. 


\section{What is an observation?}

It is the Superposition Effect as Measured (SEM) between some entities in our experiments.

- 1. We do not know completely even a single entity, whether an elementary particle or a complex system, in every detail under different conditions of interactions.

- 2. All sensors (interactants, or detector-detectee) have inherent restrictive rules of engagements with each other, which limit the observables that we can record. A detector is incapable of "reporting" to us everything that it experiences.

-3. Thus, we are for ever deprived of complete information regarding any observation even for a very well defined experiments.

- 4. So, science of modeling or mapping nature will always be a "work in progress". We are forced to organize a finite set of observations predictable by an equation that can be logically completed only by incorporating human logic supported hypotheses, some of which may or may not be congruent with the eventual cosmic logic we are seeking to discover.

- 5. A logically complete equation is also logically closed to accommodating any radically different logic. If the "successful" equation, like that of Schrödinger's, can accommodate a wide variety of observations, we may suffer from the illusion that it represents a "complete" theory!

- 6. We are thus forced to accept a dialectical contradiction in mapping nature. We must build a map only for a "local terrain" and even that has to be based on incomplete knowledge of the terrain, while our vision is to create roads to map the entire cosmic forest. Thus, all of our theories are, by definition, provisional and incomplete. Most likely they contain mistaken concepts (hypotheses) and hence cannot be simply slapped together to create one integrated cosmic map (a theory of everything).

- 7. Only choice is to advance iteratively by frequently stepping backward to identify and reject some of the human logic (hypotheses) from individual "solved" maps, which are not convertible to cosmic logic.

- 8. CC-LC-ER epistemology is the proposed tool to facilitate this iterative distilling process to steadily convert human logic into the desired cosmic logic allowing the construction of self-congruent bigger maps in multiple steps. 


\section{We are still Buddha's blind men who were instructed more than $\mathbf{2 5 0 0}$ years ago to diligently employ the CC-LC-ER epistemology to reconstruct the cosmic elephant!}
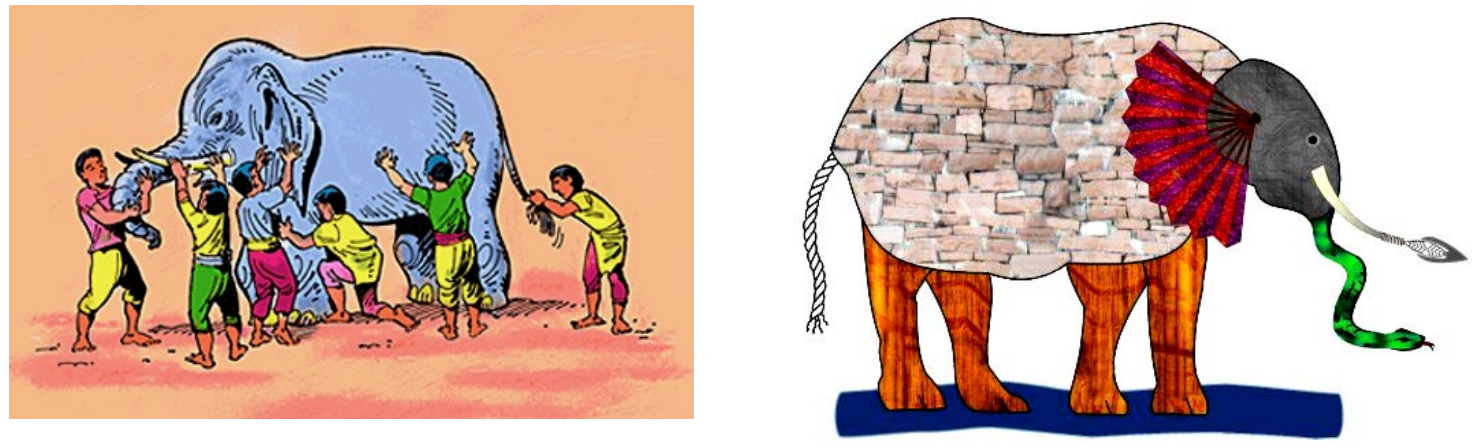

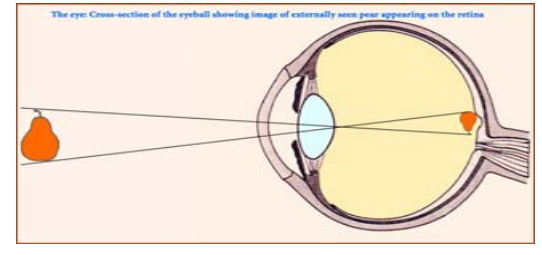

Reminder:

- We don't "see". We are blind. We manipulate \& interpret sensory chemical input.

- Barriers to our minds are self imposed, many a times collectively!

- We have found only useful "working rules", not inviolable LAWS!

- Above images are from web.
- Only by demanding conceptual continuity (CC) by imposing logical congruence (LC) among all the observations by all the blind men did they reconstruct the elephant with some semblance to reality, but outer reality only. Emergencism and reductionism (ER) are essential to appreciate the whole living elephant!

- To visualize the processes in the micro world of interactions, we must cultivate the humility to accept that we are literally blind!

- All of our information is what our sensors tell us. Like the blind men, the sensors themselves do not see \& experience everything and they are not capable of reporting everything they experience. 


\title{
Logically dissecting \\ the observation process
}

\author{
or \\ the superposition effect as measured (SEM)
}

SEM provides the foundational information to CC-LC-ER epistemology 


\section{Universality of superposition effects as measured (SEM) is at the core of doing physics}

- 1. We can scientifically measure only re-producible quantitative transformations that are experienced by our interactants (or detector-detectee, or sensor-sensee).

-2. Any transformations in measurable physical parameters requires energy exchange between the interactants.

- 3. The energy exchange must be guided by a force of interaction between the interactants and it must be strong enough to facilitate the exchange of energy, which are usually constrained by the characteristic limitations of each interactant.

-4. All force rules being distance dependent, energy exchange between the Interactants requires that they must experience each other as locally present or physically superposed entities (experience each other within their sphere of influence).

Evidence of universal "Superposition effect" (or causation steps): Interactants must be physically superposed within the range of their interacting force that will allow some energy exchange followed by some transformations that is measurable for us. Superposition effect is an active local process, not a passive mathematical principle! Mathematical formulation must recognize this Reality Epistemology. 


\section{Re-visiting superposition effects as measured (SEM) in view of the four force rules that we understand, so far!}

1. Gravitational force (GF): All cosmic entities, from galaxies, stars, planets, atoms and elementary particles, the entire observable material universes is effectively superposed on each other as far as GF is concerned. GF is weak; its range is very long.

2. Electromagnetic force (EMF): Stability of atoms, molecules and their all possible transformations, including their interactions with electromagnetic waves (light, etc.) are all dictated by this force. A dominant part of the biospheric evolution is driven by this force. EMF is relatively stronger than GF, but the range is shorter. The superposition effects due to the EMF from the molecules of two different human bodies are essentially negligible (un-entangled) on a first order analysis.

3. Weak Nuclear force (WNF): Radioactivity and related isotopic nuclear transmutations are a byproduct of this force. The range of WNF is of the order of the size of the atomic nuclei. The superposition effects due to two radioactive atomic nuclei within the same bound molecule is negligible within the first order analysis.

4. Strong nuclear force (SNF): Our slow physical evolution relies on the stability of an array of nuclei held together by this SNF, built into stable atoms and molecules by the EMF and held on the surface of the Earth under the atmosphere by the GF. The superposition effects due to two atomic nuclei within the same bound molecule is negligible within the first order analysis.

While the emergent observable material and light based universe may generically appear as NON-LOCAL, a careful analysis of SEM tells us that all measured phenomena are necessarily LOCAL since all forces of interactions have a finite physical range! Entanglement is operative within the operating range of the force. 


\section{An example of conceptual integrative power of CC-LC-ER epistemology}

We are expecting major new developments based on this over-arching understanding:

There is a preponderance and universality of harmonic undulations (internal and external),

however,

only a rare few constitute physically propagating waves! 


\section{Our universe is dominated by various harmonic undulators, but very few of them obey strict wave equation!}

Everything observable in this universe are built out of a few stable elementary particles, which are all emergent in the cosmic medium with some intrinsic internal harmonic undulation or spin. Even the complex macro systems like the Solar system and the galaxies have their own characteristic internal harmonic undulations. Even human technology is replete with mechanical and electrical harmonic undulators. Only rare few harmonic undulations are waves in reality !
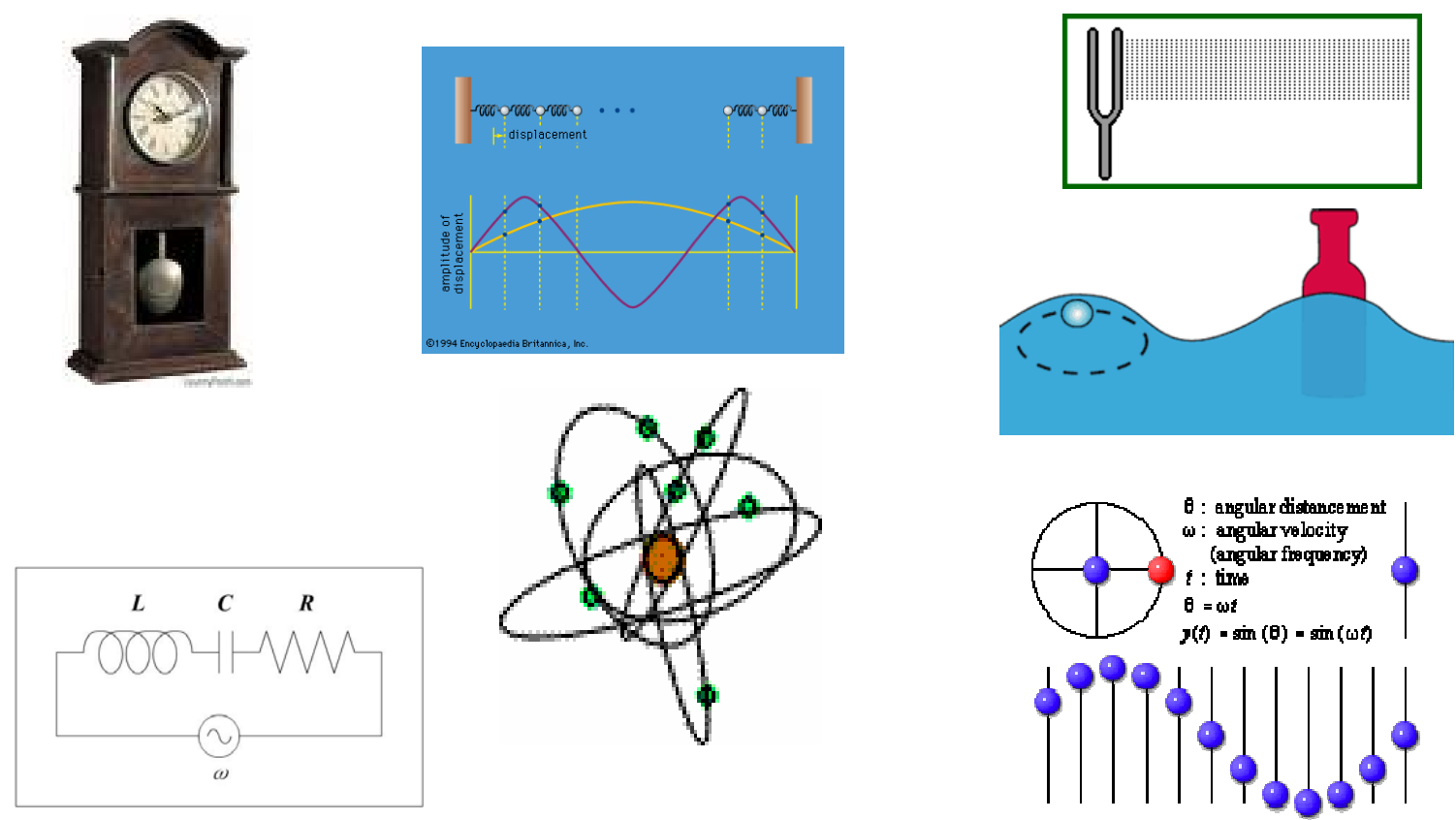

$\sin 2 \pi v t$ or $\cos 2 \pi v t$; frequency $v$ is inverse of the harmonic period $\tau$ 


\title{
We are really no longer fundamentally limited by Heisenberg's Uncertainty Relation (HUR)
}

\author{
HUR is essentially a corollary of Fourier theorem
}

\section{And the "Mother" theorem has never been elevated to the status of a principle of Physics !}

And, now our

visualization capability

has reached the atomic domain that was unimaginable during Heisenberg's time!

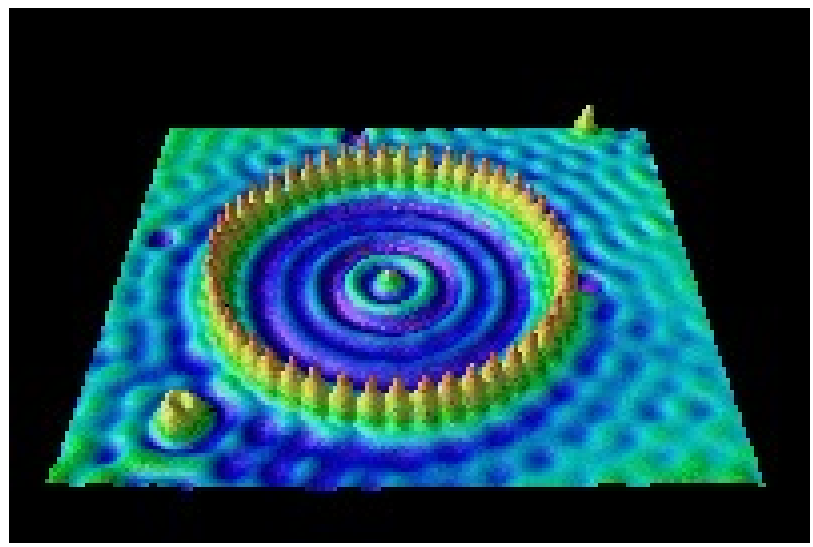

Scanning tunneling micrograph of 48 iron atoms arranged in a circle by picking and placing one atom at a time

1. By ignoring HUR, while the core achievements of QM do not suffer at all, the QM philosophy can get rid of all the unnecessarily assigned mysticisms like non-causality, non-locality, teleportation, etc. in measurements that defies our SEM process as we have defined.

2. Does the structure of Schrödinger's "wave equation" really represent a propagating wave? A propagating wave require second derivative of time equated to second derivative of space coordinate.

3. Are elementary particles and atoms really non-local and "dispersive" probability waves? Then how could we manipulate Angstrom size individual atoms using modern nano-tipped tools?

4. Etc., etc. 


\section{Another example of application of CC-LC-ER epistemology in compliance with SEM:}

We discover that we have been missing something rather fundamentally simple for millennia!

- Light beams by themselves cannot produce interference effects !

- Alternately speaking, the superposition effects as measured (SEM) due to light beams can become observable only when some interacting material dipoles are simultaneously stimulated by all the superposed beams on them.

Interpretational and technological implications are enormous ! 


\section{Examples of absence of superposition effects from light beams in the absence interacting material dipoles!}

Trillions of unwanted beams can cross the path of our desired light beam before we can receive and analyze the un-modified properties carried by the beam.

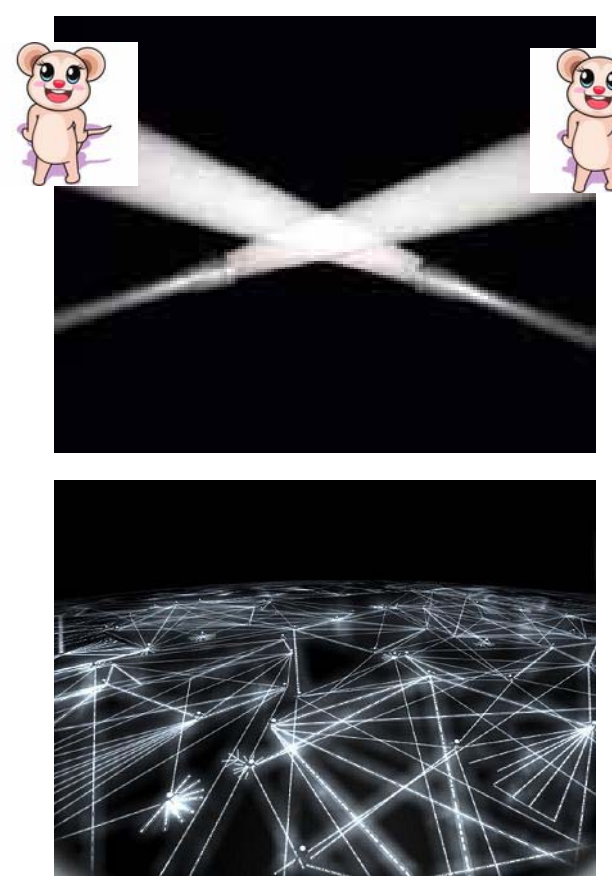

\section{Otherwise}

The visual world would have been full of spatial and temporal scintillations (speckles).

WDM internet data would have been destroyed by temporal interference (heterodyne effect).

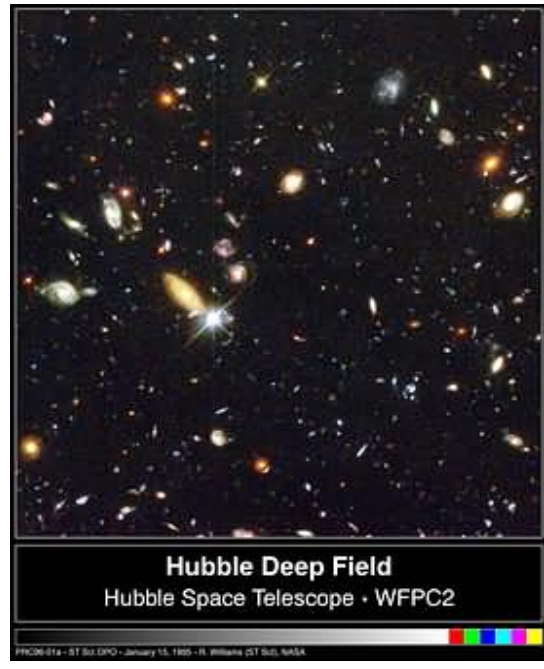

Expanding universe, indicated by Doppler shift, would not have been measurable.

Well formed light beams cross each other without modifying each others spatial and/or temporal energy distribution. 


\section{Light and sound beams pass through each other essentially undistorted from one corner to another in a crowded stadium, unperturbed by millions of other beams!}

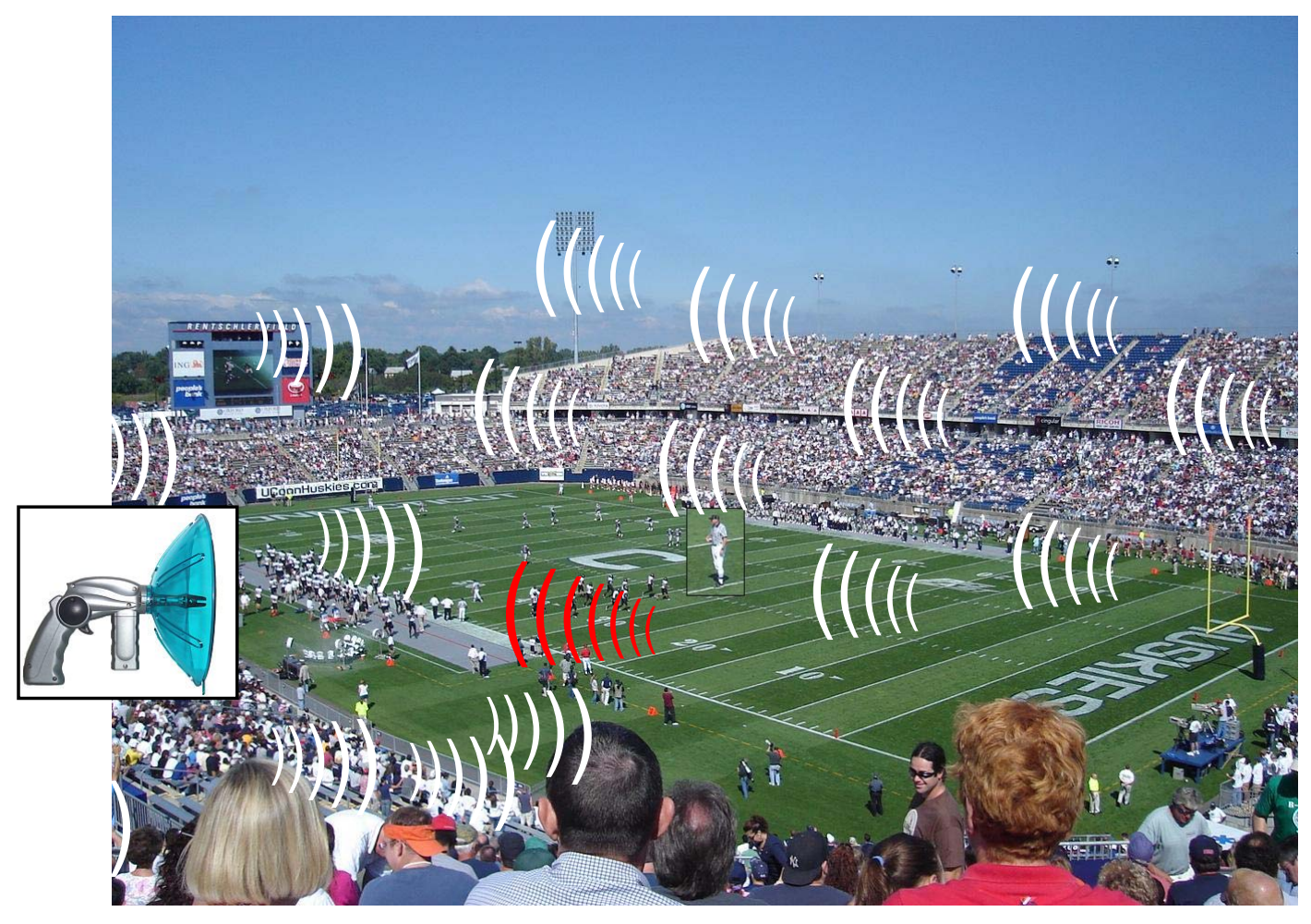

C. Roychoudhuri, SPIE Newsroom: http://newsroom.spie.org/x5251.xml 


\section{Even water waves pass through each other without perturbing each others collective behavior except at the locations of actual superposition!}
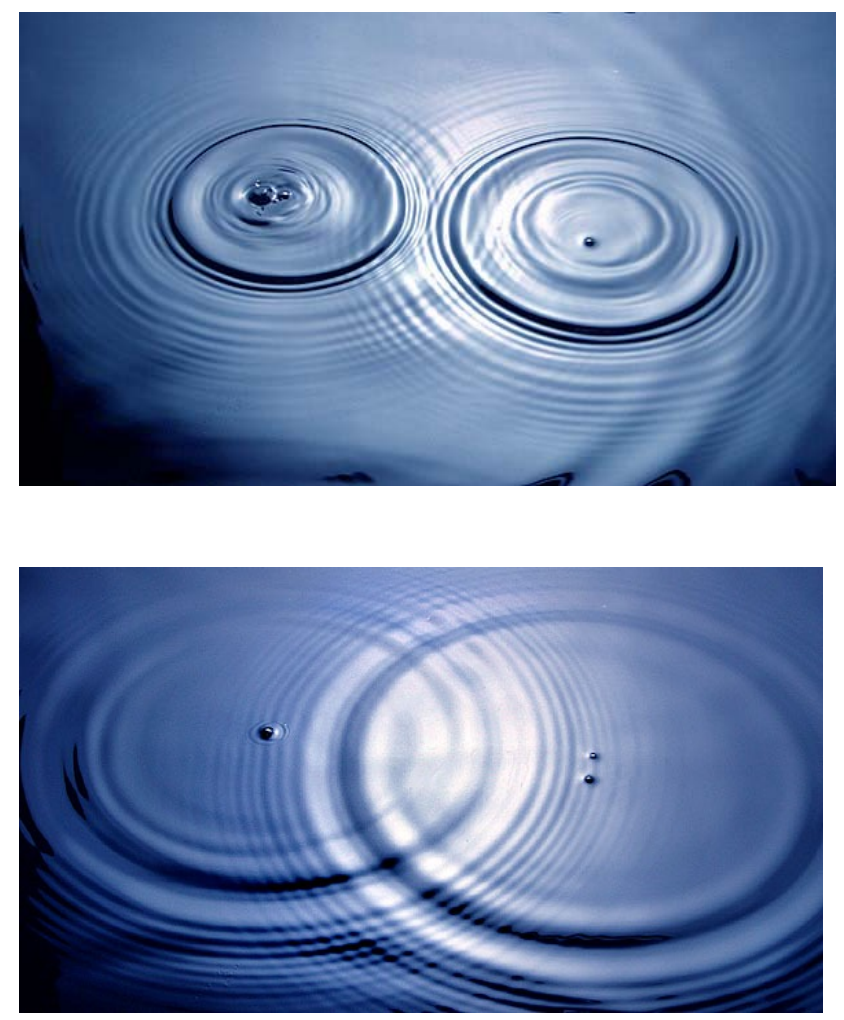

- Harmonic undulations on a water surface and their propagation remains undistorted, even though the water in the LOCAL-regions of superposition show the modified resultant undulations.

- Wave forms by themselves do not interfere. If the supporting medium that is observable and shows local superposition effects.

- Unfortunately the cosmic medium in which light is emergent is not yet directly observable to us to display "local interference" of light beams.

Non-interference of wave forms is a generic property of nature! 


\section{An example of productivity of CC-LC-ER epistemology}

Next two slides provide a list of recent publication by the author based on CC-LC-ER epistemology \& non-interference of light

\section{Notice:}

- The titles themselves are indicative of the epistemology used.

- The productivity in terms of topic diversity within a period of four years (2003-2007) 


\section{List of recent publication by the author based on CC-LC-ER epistemology \& non-interference of light}

1. "Shall we climb on the shoulders of the giants to extend the REALITY horizon of Physics?" by C. Roychoudhuri, invited talk at the 4th International Conference on "Quantum Theory-Foundational Reconsiderations", at Vaxjo U., Sweden, Jun.11-16, 2007; to be published in 2007.

2. "Can classical optical superposition principle get us out of quantum mysticism of non-locality and bring back REALITY to modern physics?" by C. Roychoudhuri, invited talk at the ETOP conference at Toronto, Jun.3-5, 2007; to be published in 2007 by SPIE;

3. The Nature of Light: What Is a Photon? by C. Roychoudhuri, A. F. Kracklauer \& Kathy Creath,; CRC Press (2007); in preparation.

4. "Can a deeper understanding of the measured behavior of light remove wave-particle duality?" by C. Roychoudhuri, SPIE Proc.Vol.6664, paper \#2 (to be published, Aug. 2007).

5. "Can we get any better information about the nature of light by comparing radio and light wave detection processes?" by C. Roychoudhuri and P. Poulos, SPIE Proc.Vol.6664, paper \#12 (to be published, Aug. 2007).

6. "Can the hypothesis 'photon interferes only with itself' be reconciled with superposition of light from multiple beams or sources?" by C. Roychoudhuri, N. Prasad and Q. Peng, SPIE Proc.Vol.6664, paper \#24 (to be published, Aug. 2007).

7. "Bi-centenary of successes of Fourier theorem! Its power and limitations in optical system designs" by C. Roychoudhuri, invited paper, Proc. SPIE Vol. 6667, paper \#18 (Oct. 2007).

8. "If EM fields do not operate on each other, why do we need many modes and large gain bandwidth to generate short pulses?" by C. Roychoudhuri, N. Tirfessa, C. Kelley \& R. Crudo,; SPIE Proceedings, Vol. 6468, paper \#53 (2007).

9. "Locality of superposition principle is dictated by detection processes" by C. Roychoudhuri, Phys. Essays 19 (3), September 2006.

10. "Spectral Super-Resolution by Understanding Superposition Principle \& Detection Processes", by C. Roychoudhuri and M. Tayahi, Intern. J. of Microwave and Optics Tech., July 2006; manuscript ID\# IJMOT-2006-5-46: http://www.jjmot.com/papers/papermain.asp.

11. "Various ambiguities in re-constructing laser pulse parameters" by C. Roychoudhuri and N. Prasad, proceedings of the October, 2006 IEEE-LEOS Annual Conference, Montreal, Canada; invited.

12. "Do we count indivisible photons or discrete quantum events experienced by detectors?" by C. Roychoudhuri and N. Tirfessa, Proc. SPIE Vol.6372-29 (2006). 


\section{List of recent publication by the author based on CC-LC-ER epistemology \& non-interference of light (list-p.2)}

13. "If EM fields do not operate on each other, how do we generate and manipulate laser pulses?" by $C$. Roychoudhuri, D. Lee and P. Poulos, Proc. SPIE Vol.6290-02 (2006).

14. "Are dark fringe locations devoid of energy of superposed fields?" by C. Roychoudhuri and C. V. Seaver, Proc. SPIE Vol. 6285-01 (2006), invited.

15. "A critical look at the source characteristics used for time varying fringe interferometry" by $\mathrm{C}$. Roychoudhuri and $\mathrm{N}$. Tirfessa, Proc. SPIE Vol.6292-01, (2006), invited.

16. "Role of the retinal detector array in perceiving the superposition effects of light" by C. Roychoudhuri and V. Lakshminarayanan, Proc. SPIE Vol.6285-08 (2006).

17. "Reality of superposition principle and autocorrelation function for short pulses" by C. Roychoudhuri, Proc. SPIE Vol. 6108-50 (2006).

18. "If superposed light beams do not re-distribute each others energy in the absence of detectors (material dipoles), can an indivisible single photon interfere by/with itself?" by C. 18. Roychoudhuri, SPIE Conf. Proc. 5866, pp.26-35 (2005).

19. "If superposed light beams do not re-distribute each others energy in the absence of detectors (material dipoles), can an indivisible single photon interfere by/with itself?" by C. Roychoudhuri, Proc. SPIE Vol.5866, pp.26-35 (2005). 20. "The Nature of Light: What Is a Photon? Eds. C. Roychoudhuri, Katherine Creath and A. F. Kracklauer, Proc. SPIE Vol.5866 (2005); Year of Einstein Special Conference.

21. "Propagating Fourier frequencies vs. carrier frequency of a pulse through spectrometers and other media" by C. Roychoudhuri, Proc. SPIE Vol.5531, 450-461(2004).

22. The Nature of Light: What is a Photon?", Guest Eds. C. Roychoudhuri \& R. Roy, Optics \& Photonics News Trends; special issue of OPN, October 2003. [http://www.osa-opn.org/abstract.cfm?URI=OPN-14-10-49].

23. "Measuring properties of superposed light beams carrying different frequencies" by D. Lee and C. Roychoudhuri, Optics Express 11(8), 944-51, (2003), [http://www.opticsexpress.org/abstract.cfm?URI=OPEX-11-8-944].

24. "Limits of DWDM with gratings and Fabry-Perots and alternate solutions" by C. Roychoudhuri, D. Lee, Y. Jiang, S. Kittaka, M. Nara, V. Serikov and M. Oikawa, Proc. SPIE Vol.5246, 333-344, (2003), invited. 


\title{
Summary of the results expressed in the published papers listed before that provide both improved understanding of classical and quantum optics and potential for a wide variety of technological innovations
}

\begin{abstract}
- 1. Superposition effects are created by detectors: (i) Accordingly changing the detectors or modifying their physical properties by secondary external fields will change the out come due to the same set of superposed optical fields. (ii) The definition and the theory of coherence need to be expressed in terms of correlation of multiple dipole undulations of the same detector induced simultaneously by multiple fields rather than as field-field correlations.

Consequently new and more accurate theory and measurement techniques can be developed for characterizing very short pulses.
\end{abstract}

-2. Optical signals and responding material dipoles are all space, time and energy

finite: (i) Generalized theory of spectrometry based on finite pulses conform to classical formulas for time integrated records, but time-frequency band width product (spectral resolution) is not a fundamental limit of nature but a function of our sensor arrangements. (ii) Pulse broadening through "dispersive" media is due to "time diffraction" just as space-finite aperture introduce fringe broadening.

\section{- 3. Light beams by themselves do not produce mutual energy re-distribution} (interference fringes) either in space or in time: (i) Mode-lock laser theory needs to be modified in terms of properties of saturable absorbers (interacting dipoles) leading to more innovations in short pulse laser technology. (ii) The concept of 'photons interfere only with themselves' (Dirac) needs to be fundamentally revised, which will open up newer and better way of creating possible practical quantum computers, etc. 


\section{It is time to frame great new questions to generate great new break through knowledge about the ongoing processes in nature}

- Great questions of any time are framed based on existing observations. If the answer is right, it will necessarily lead to new observations and hence new questions beyond the original question.

- Biologists, as humble reverse-engineers, diligently emulating the chemical processes behind all living activities, have found an over-arching and integrating working principle - the DNA helix - across all living things!

- Physicists have been happily playing with almost half a dozen separate "solved puzzles" and trying to force fit them together for over half a century even though they are not logically self-congruent for the desired merger.

We have proposed a great new CC-LC-ER epistemology that can accommodate great questions from great minds allowing iterative distillation of many untenable human logic from many of our separate theories to increasingly higher planes of mutual congruence \& integratability towards cosmic logic and hence cosmic reality even though we can only start with incomplete and mutually incongruent theories! 


\section{Thank you for you patience!}

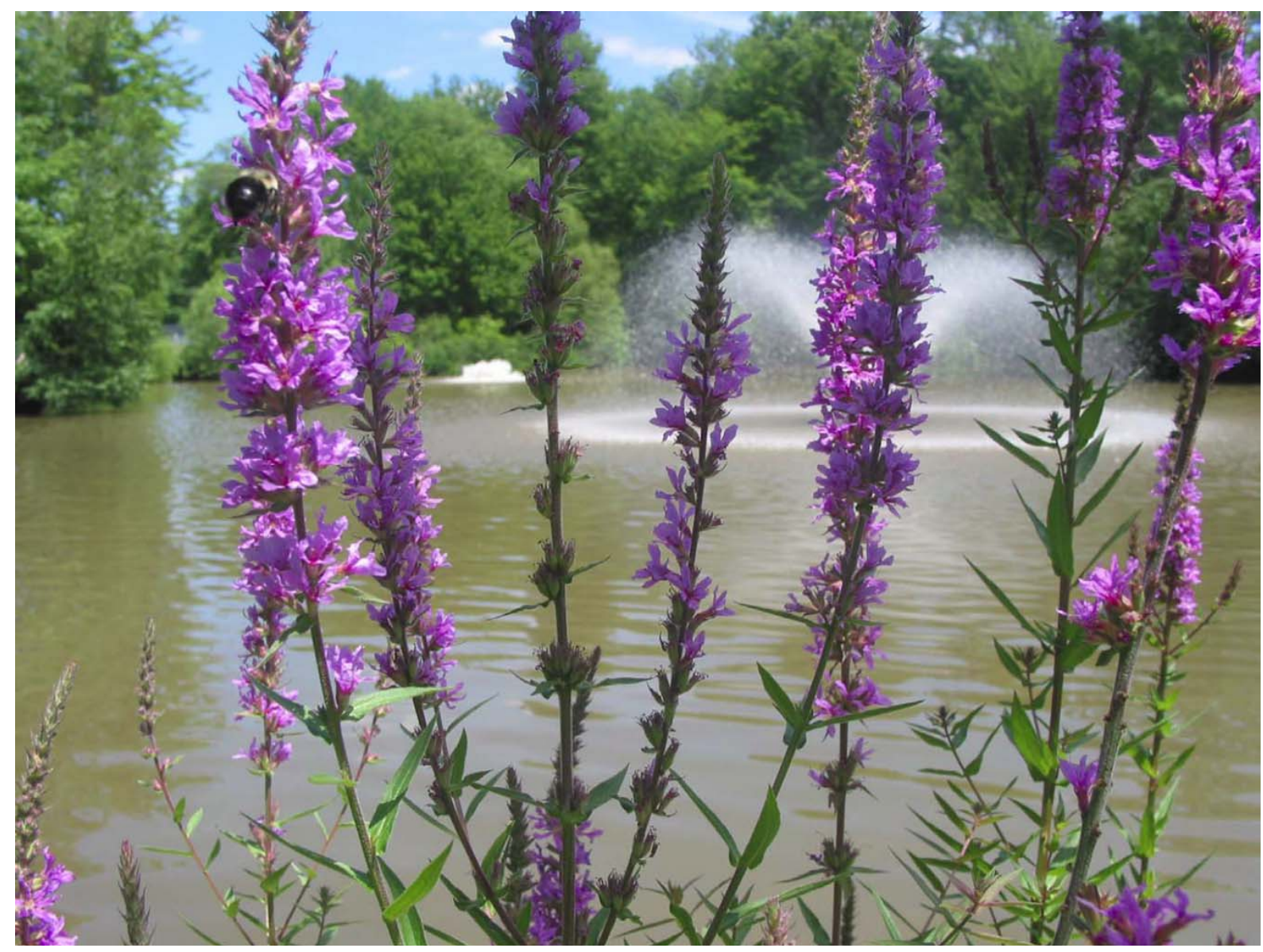

Elizabeth Park, Harford, Connecticut. By CR. 